\title{
Application of Mobile Big Data and Artificial Intelligence in the Efficiency of E-Commerce Industry
}

\author{
Hongkai Cui, ${ }^{1,2}$ Lina Xiao ${ }^{(D)}{ }^{3}$ and Xiaheng Zhang ${ }^{4}$ \\ ${ }^{1}$ School of Economics and Management, Chang'an University, Xi'an 710064, Shaanxi, China \\ ${ }^{2}$ Department of Transportation of Shaanxi Province, Xi'an 710075, Shaanxi, China \\ ${ }^{3}$ School of Management, Wuhan Donghu University, Wuhan 430000, Hubei, China \\ ${ }^{4}$ College of Business and Trade, Nanchang Institute of Science and Technology, Nanchang 330108, Jiangxi, China \\ Correspondence should be addressed to Lina Xiao; xiaoln@wdu.edu.cn
}

Received 7 June 2021; Revised 27 July 2021; Accepted 9 August 2021; Published 25 August 2021

Academic Editor: Sang-Bing Tsai

Copyright ( $\odot 2021$ Hongkai Cui et al. This is an open access article distributed under the Creative Commons Attribution License, which permits unrestricted use, distribution, and reproduction in any medium, provided the original work is properly cited.

\begin{abstract}
In recent years, with the rapid development of network technology and mobile clients, the upsurge of online shopping users has been accelerated. The development of e-commerce has become a key factor affecting the economy. At the same time, in the process of online shopping, users produce a large number of user access data and transaction data, which seem messy but contain huge commercial value. E-commerce urgently needs to mine its own data information and related data so as to obtain competitive advantage. Web data mining technology has become the focus of research in universities and enterprises. This paper summarizes these two methods and explains their advantages and disadvantages, respectively. Next, based on the advantages and disadvantages of model control and logic analysis, a logic analysis method based on model control is proposed. A formal analysis method combining finite machine and logic is proposed. This new method can analyze the responsibility, fairness, and timeliness of e-commerce protocol, provide a state transition diagram describing the working principle of the protocol, and fully analyze the responsibilities of all parties when repeated attacks occur. Experiments show that the efficiency of e-commerce is improved by $30 \%$. As mentioned above, this paper hopes to play a certain role in the development of e-commerce. This paper summarizes the time series of e-commerce data, sales changes, and the sensitivity of consumer goods, which is conducive to the prediction and evaluation of the market, seize the market trend, and provide better decision-making.
\end{abstract}

\section{Introduction}

E-commerce is a new economic activity that has emerged with the development of network and communication technology. In short, this basic concept is the process of developing business based on information technology [1]. Virtualization, low transaction cost, information interaction, high efficiency, flexible use, and transaction transparency are the characteristics of e-commerce. At the same time, it improves the potential consumer skills, expands the consumer group, accelerates the consumption demand, provides high-quality services, helps the manufacturers reduce the inventory level, accelerates the capital flow, and creates new opportunities [2]. Employment is the electronic development project exhibited by all superior companies.
Zhai Li believed the importance of analyzing the establishment of an agricultural network marketing system, and the main factors restricting the development of agricultural network market were discussed. And continuous improvement of agricultural marketing network construction to achieve the purpose of agricultural marketing network-related measures was discussed [3]. Product manager provides related network marketing system optimized information, a report on further promoting the sustainable growth of China's agriculture. However, without actual data support, the theory is not perfect [4]. Teng et al. believed that the state will successively announce Internet+ and new rural construction, as well as supply-side reform policies, which will greatly help the development of my country's rural e-commerce. However, rural Taobao only changed rural sales methods, products, and services. Starting 
from the development of my country's rural Taobao, the development trend of rural e-commerce is analyzed and promoted, and agricultural information infrastructure, e-commerce talents, agricultural product quality monitoring, and agricultural logistics issues are developed; my country's current rural e-commerce development strategy is based on dynamic fuzzy logic application, but there are still many practical problems that need to be improved [5]. $\mathrm{Wu}$ and $\mathrm{Yu}$ believed that e-commerce systems and fast logistics systems are considered two large and complex economic systems. Using the principle of cooperative order parameters, a coordinated development model of e-commerce system and fast logistics system is established to measure the degree of coordinated development and implementation in the past ten years. Research shows that the growth rate of e-commerce is higher than that of the fast logistics industry, which is consistent with the actual growth. However, they ignored the society's view of e-commerce, and the experiment was not completed [6].

The innovation of this paper is to combine dynamic fuzzy logic theory with the e-commerce system, and for the e-commerce system, this paper combines dynamic fuzzy logic theory with the e-commerce system and researches on data with dynamic fuzziness in the e-commerce system. The application of dynamic fuzzy logic technology to realize the intelligent judgment of the data in the system and the automatic selection of conditions have found a new solution for the dynamic fuzzy data processing in the e-commerce system and further the research content of the e-commerce system is enriched $[7,8]$.

\section{E-Commerce Industry Efficiency Experiment Method}

2.1. Research Methods of E-Commerce Industry Efficiency. "Research Method" is a well-designed and well-organized method that reveals the history, reality, internal logic, and evolutionary laws of the research object. This article combines e-commerce and dynamic fuzzy logic, and the specific research methods used are as follows:

(1) Literature Research $[9,10]$. According to the research needs of the thesis, in the CNKI and Bailian academic libraries, using "material selection method," "dynamic fuzzy logic," and "e-commerce" as keywords, there are more than 200 related research papers, including more than 40 doctoral and master's theses. We should carefully study the previous research results to provide important theoretical support and research ideas for this study and learn in detail the current research status and development prospects of the research.

(2) Research Method [11]. This method is widely used in academic research to obtain true and reliable information from practice, and the academic research conducted is objective and convincing. Only through thorough research and understanding of the actual situation of my country's e-commerce service companies can we conduct research on this basis, play a role, and better control our production and life.
(3) Comparative Research Methods [12]. In order to check the development status of e-commerce, it is also necessary to understand the comparative status of foreign countries in order to discover personalities and similarities and learn from each other.

(4) Employee Survey Method. Before the writing of this article, a data survey was conducted. The author also surveyed relevant personnel in questionnaires and conducted dialogues and interviews so as to have a deeper understanding of the actual situation of the research objects in this article. Respondents include service industry leaders in specific fields, such as relevant experts and employees of company e-commerce operations. Through communication with them, the research ideas of this article have been broadened, a general design concept has been developed, and the research scope has been further expanded $[13,14]$.

2.2. Experimental Methods of E-Commerce System Software. The features of this e-commerce system software are as follows:

(1) This system software is based on the browser/server structure. On the client side, you can log in to the system software for operation only through the browser. The maintenance and upgrade management of the system are centralized on the server so that it is easy to deploy and use.

(2) The operation of the system software is simple and convenient, with a friendly interface and flexible operation.

(3) The structure and operation process of the system are consistent with the actual business process, and the simulation is strong, which is suitable for the basic platform for theoretical research.

(4) The data structure design is reasonable, the roles imitated by the operator in the system are intertwined, and at the same time, it is flexible and changeable when processing, which is more convenient for the operator to clarify ideas and understand the process.

(5) The system has a complete structure and complete functional modules. There are not only online transaction modules but also online office modules, network operation modules, and e-commerce application modules. The content of the experiment makes the experimental structure more reasonable and ensures the comprehensiveness of the content and the advancement of knowledge.

\section{Correlation Experiment of Dynamic Fuzzy Logic}

$Q(x, x) * P(x, x)$ is the basic form of DFL rules, and $Q(y, y)$ and $P(y, y)$ are the antecedents and corollaries of dynamic fuzzy logic reasoning $[15,16]$. From the dynamic fuzzy 
knowledge representation method in Chapter 2, the premise and conclusion form of complex meaning can be expressed easily. However, for convenience, it is simplified as follows:

$$
Q_{1}(\overleftarrow{x}, \vec{x}) \longleftrightarrow Q_{2}\left(\overleftarrow{x}_{2}, \vec{x}_{2}\right)
$$

After deduction, the final result is as follows $[17,18]$ :

$$
W_{1}(\overleftarrow{x}, \vec{x}) \cup Q_{2}\left(\overleftarrow{x}_{2}, \vec{x}_{2}\right) \cap W_{1}(\overleftarrow{x}, \vec{x}) \cup Q_{2}\left(\overleftarrow{x}_{2}, \vec{x}_{2}\right)
$$

Containment rules are defined as follows:

$$
(x, x), \partial а A_{\alpha}(x, x) \longrightarrow C(x, x) .
$$

A rule refers to a dynamic fuzzy rule covering each example. It can be composed of relevant examples and irrelevant examples. When the rules are matched, irrelevant examples can be removed $[19,20]$.

The approximation rules are defined as follows:

$$
\text { “ }(x, x), " a B_{\alpha}(x, x) \longrightarrow C_{\alpha}(x, x) .
$$

The rule expression $(x, x)$ is stronger than $B$, and $(x, x)$ is more subordinate to $C$ (because they are simulated by conditional constraints) [21, 22].

The limit rules are defined as follows:

$$
\text { “ }(x, x), " a D_{\alpha}(x, x) \longrightarrow C_{1-\alpha}(x, x) .
$$

It is now verified that if the sender A obtains the EOR defined in the agreement, it can prove that the receiver is nonrepudiated. The analysis is as follows: from the known,

$$
A \longrightarrow(B \longrightarrow h(r)) \cap B \in r .
$$

The message sender $A$ can prove that the message receiver $\mathrm{B}$ is responsible for $h(m)$ and $B$ owns $m$. After checking the consistency of $h(m)$ and $m$, we can get

$$
S_{a} \in\left\{\{h(m)\} k_{b}^{-1}\right\} k_{\mathrm{jtp}}^{-1}
$$

This step will verify whether it can ensure that $A$ and $B$ obtain the corresponding evidence when reaching the final state on the normal execution path of the state transition diagram:

$$
\text { EOO } \in S_{B}^{3}=W_{1}-h(m) .
$$

The initiator of event $e$ is $B$, so suppose that participant $A$ is an attacker or an external attacker $C, A$ or $C$ intercepts the message $M$ sent by $B$ to TTP [25], then pretends to be $B$ and sends message $M$ to TTP, and then intercepts from the message sent by TTP to $B$ so that $A$ can get EOR, $C$ can get EOO, but $B$ cannot get EOO $[26,27]$. Therefore, this protocol may be attacked by replay. According to the analysis results of the above three small steps, the protocol meets the initial fairness but does not meet the time limit and may be attacked by regeneration, so the protocol does not meet the fairness. In the end, it can be obtained that the CMP1 protocol meets the accountability but does not meet the fairness and time limit, and there is the possibility of replay attacks [28].

\section{Example Analysis of Dynamic Fuzzy Logic in the E-Commerce Industry}

Based on the relevant theoretical knowledge obtained in the previous section, the following examples are used for easyto-understand explanations.

Example 1. An enterprise operates an e-commerce business, and its sales products include agricultural products and car modification parts. Agricultural product sales revenue $(h 1)$ is related to purchase cost $(D)$ and climate $(B)$; the sales profit of car modification parts $(h 2)$ is related to the sales volume of car modification parts $(C)$ and the cost of car modification parts $(E)$; income $(A)$ is determined by the profit from the sale of agricultural products and the profit from the sale of modified car parts. At the end of 2006, the general manager obtained the company's annual income through the e-commerce system year-end summary table. This year's income is 9 million yuan and 500,000 yuan less than the average income in previous years. After reading the year-end summary, the general manager wanted to find out the reasons for the low income this year. Through investigation and analysis, the e-commerce system feedbacks the following news:

(1) The climate this year is worse than that in previous years;

(2) The sales volume of automodified parts is more than in previous years;

(3) The product cost of car modification parts has not been reduced;

(4) The purchase cost of agricultural products has not been reduced; the reasoning process to solve this problem is as follows: first, the problem is formalized, and the factors $A, B, C, D$, and $E$ in the problem are regarded as shown in Table 1.

According to the results, it can be judged that U1's sales are evaluated as hot, but sales will decrease in the future; U2's sales are evaluated as best-selling, and sales will be better in the future; U3's sales evaluation is unsalable, it will not improve in the future, so it is recommended not to buy. The sales evaluation of $\mathrm{U} 4$ is average, but it will be worse and worse in the future, and it is recommended to reduce the price; U5 sales evaluation is general, but sales will increase in the future.

As shown in Figure 1, the Internet network resource counter includes the number of IP addresses, the number of domain names, the number of websites, and the international broadband export bandwidth. The China Network Information Center (CNNIC) released its 39th "Statistical Report on China's Internet and Network Development," which shows the changes in various indicators in the past year. As shown in Table 2, in January 2017, there were 338 million IPv4 addresses, 21188 IPv6 addresses/324,227 
TABLE 1: Table of propositional variables.

\begin{tabular}{|c|c|c|c|c|}
\hline & 0 & 1 & $Z$ & $M$ \\
\hline Income & Low & High & Increase & Cut back \\
\hline Climate & Normal & $\mathrm{Bad}$ & Deterioration & Get better \\
\hline Sales volume of modified car parts & Low & High & Increase & Reduce \\
\hline Purchase cost of agricultural products & Less & Many & Increase & Cut back \\
\hline Cost of modified car parts & High & Low & Reduce & Improve \\
\hline
\end{tabular}

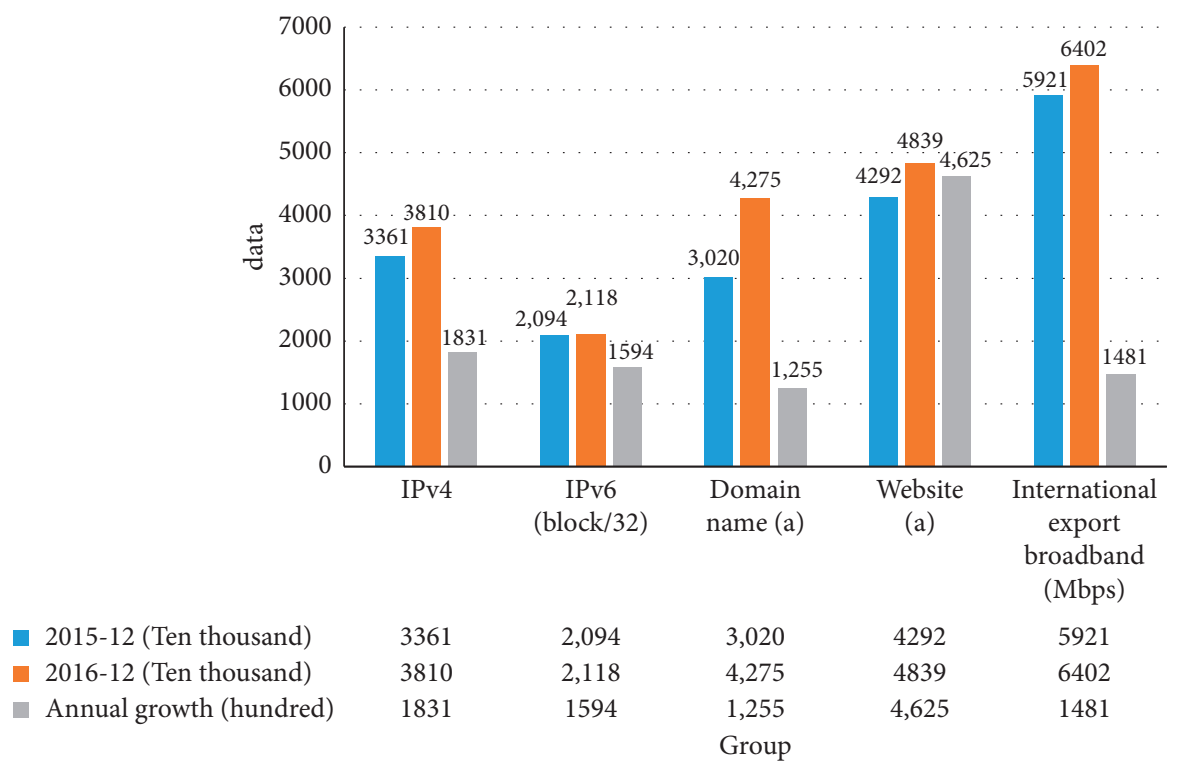

Figure 1: Comparison of basic internet resources in China from December 2015 to December 2016.

TABLE 2: Comparison of changes in inventory and propositional variables.

\begin{tabular}{lcccc}
\hline & 0 & 1 & $X$ & $Y$ \\
\hline Commodity inventory A & No & Have & Increase & Cut back \\
Brand awareness B & Unknown & Famous brand & Rise & Decline \\
Sales status C & Low & High & Rise & Decline \\
Temperature D & Cold & Hot & Rise & Decline \\
Holiday E & Yes & No & Close to & Already far away \\
\hline
\end{tabular}

domain names, a total of 4.82 million websites, 6 international export bandwidths, and 64 million megabits per second, indicating that China has abundant Internet resources that are conducive to the development of e-commerce especially domain names. Since January 2017, domain names have increased by $36.3 \%$ year-on-year and IPv4 has increased by $0.5 \%$ year-on-year. IPv6 (block/32) has increased by $2.9 \%$ compared with the last year. The above data show that China currently has a high demand for basic Internet resources and continues to strengthen its Internet infrastructure to lay the foundation for the development of e-commerce.

As shown in Figure 2, 45 high-traffic mobile e-commerce keywords are closely related. The lines in the figure link two linkable keywords. You can search for social network functions on the keyword network map, for example, search for the central position of high-frequency keywords in e-commerce and search for bibliographic hot spots of mobile e-commerce according to the scope of e-commerce. The main content of domestic scholars in mobile e-commerce business model research covers the basic theoretical concepts of business models, technical support, application research, types of business models, and other important aspects. The business model of mobile e-commerce has evolved over time. It is tailored to business development goals and gradually improved through experience summaries. At the same time, the business model is accompanied by the mutual cooperation and common development of major participants in the retail industry value chain, gradually forming a business profit model.

As can be seen from Table 2, among them, 0 means that there is no stock in the existing inventory of a product that is being sold; first, there are not enough items in the inventory of the goods being sold. $X$ means that the products currently on sale are in the best-selling period. $X$ means that it is in this stage, the goods sold are in the stage of market shrinkage and it is not good to purchase, and $x, x \in(0,1)$. Assume that the existing Haier home air conditioner: DC high-efficiency dual 


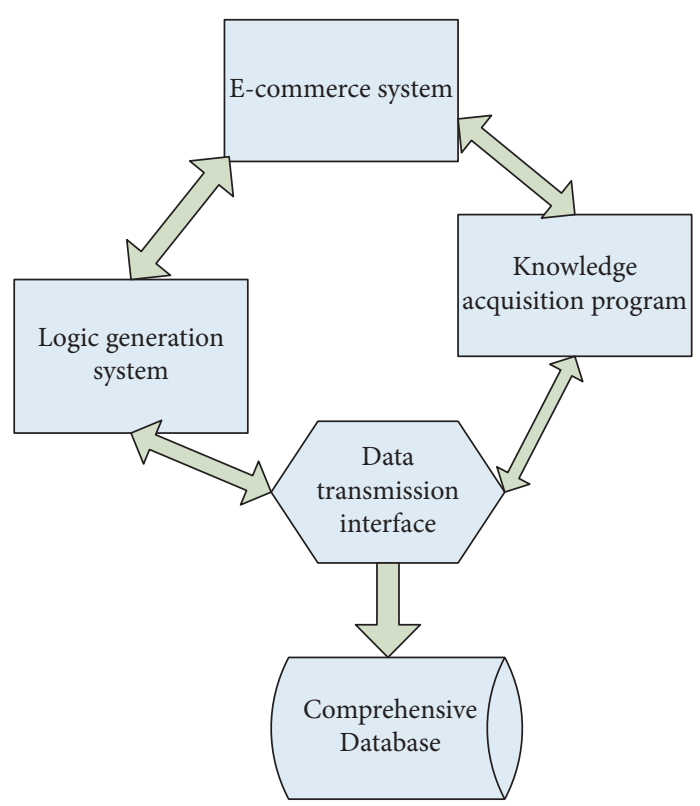

FIGURE 2: Network diagram of high-frequency keywords based on the subject area of mobile e-commerce.

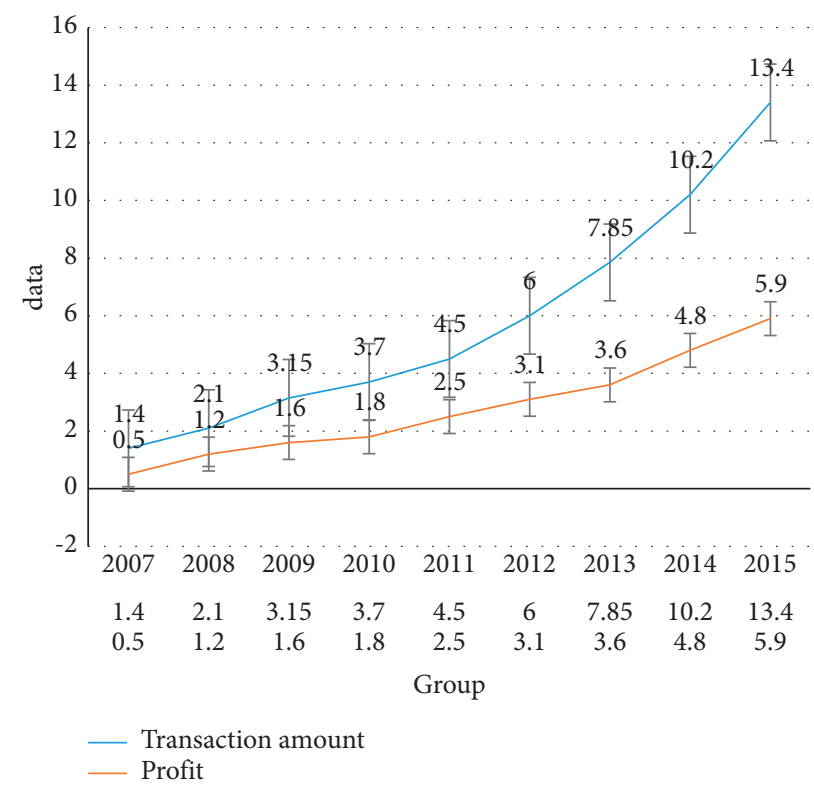

Figure 3: 2011-2015 China's e-commerce market transaction scale.

fresh air, model: KFR-35GW/V (DBPZXF), the current status is in stock, 2 pieces were sold the day before, the weather is clear today, and the National Day has just passed, and it is a promotion in the end of the period and can be obtained from 13 known conditions: $P$ \{ Inventory of existing products, well-known brands, sales are in decline, the weather is clear, just past the National Day, the end of the promotion period\}, and we can get the rule set PS from the relevant purchase rules of $\mathrm{KB}$.

As shown in Figure 3, from January 2016 to September 2016, the scale of online shopping transactions in my country was 20.2 trillion yuan, an increase of 3.68 trillion yuan over the previous year and an increase of $22.3 \%$, which is 2.14 times the growth rate of transaction volume. During the same period, the total retail sales of consumer goods and the scale of e-commerce transactions grew rapidly from 2007 to 2015 , from 1.4 trillion in 2007 to 13.4 trillion in 2015, with an average growth rate of $33.5 \%$. The rapid development of e-commerce is inseparable from the support of national policies and the acceleration of structural reforms on the supply side.

\section{Conclusions}

This paper takes the application of mobile big data and artificial intelligence in the efficiency of e-commerce 
industry as the research object and uses the theoretical knowledge of fuzzy logic dynamics. First, a method for representing dynamic fuzzy data is provided, dynamic fuzzy output. Next, we apply these basic theories to an e-commerce system, design and implement an example system, and study the personalized recommendation process of mobile e-commerce. In addition, we also study the data mining algorithm and recommendation for mobile e-commerce. The research results of this paper further enrich the research content of the e-commerce system. The business model can adapt to the development trend of modern technology, but due to the lack of information and data collection, the business model may be incomplete. Artificial intelligence provides a good environment for making strategy and decision and provides data environment and decision analysis support for the specific application system. When facing the specific strategy and decision-making problem of specific application, AI starts from data preparation, first establishes or virtualizes an integrated data, and then uses scientific decision analysis tools in the integrated data environment to support strategy and decision-making through data analysis, knowledge discovery, and other processes. Finally, explain, implement, and analyze it to get the final result. In the whole process, integrated data environment and decision analysis tools are very important and indispensable.

\section{Data Availability}

No data were used to support this study.

\section{Conflicts of Interest}

The authors declare that there are no conflicts of interest with any financial organizations regarding the material reported in this manuscript.

\section{Acknowledgments}

This work was supported by Jiangxi Provincial Department of Education Science and Technology Research Project "Jiangxi Province Cross Border E-Commerce Industry Chain Cluster Research" (GJJ202505), Nanchang Institute of Science and Technology Introduced Talents Research Start Project "Cross Border E-Commerce Industry Chain Cluster Research" (NGRCZX-20-11), and the grants from Hubei Provincial Collaborative Innovation Centre of Agricultural E-Commerce (Wuhan Donghu University Research [2019] No. 17 Document).

\section{References}

[1] J. Peng, J. Quan, and L. Peng, "It application maturity, management institutional capability and process management capability," Journal of Organizational and End User Computing, vol. 31, no. 1, pp. 61-85, 2019.

[2] S. Ivanaj, G.-B. Nganmini, and A. Antoine, "Measuring E-learners' perceptions of service quality," Journal of Organizational and End User Computing, vol. 31, no. 2, pp. 83-104, 2019.
[3] I. Kitouni, D. Benmerzoug, and F. Lezzar, "Smart agricultural enterprise system based on integration of internet of things and agent technology," Journal of Organizational and End User Computing, vol. 30, no. 4, pp. 64-82, 2018.

[4] Z. Li, "Research on the construction mode of agricultural products network marketing system in the E-commerce era," Economic Research Guide, vol. 19, pp. 48-49, 2018.

[5] X. Teng, S. He, H. Ma et al., "Research on the development of rural E-commerce in my country," Logistics Engineering and Management, vol. 38, no. 4, pp. 117-119, 2016.

[6] S. Wu and B. Yu, "Research on the collaborative development path of e-commerce and express logistics," Management Review, vol. 28, no. 7, pp. 93-101, 2016.

[7] Z. Xiao, "Research on price competition between e-commerce retailers and traditional retailers," Economics, vol. 7, p. 00146, 2016.

[8] T. Jia, L. Liang, and D. Yan, "Current status and operation mode of cross-border e-commerce logistics in my country," Modern Economic Information, vol. 15, p. 335, 2018.

[9] T. Luo, "Analysis on the development of China's agricultural product e-commerce," Business Stories, vol. 32, pp. 124-125, 2016.

[10] F. Su, "The current situation and operation mode of my country's cross-border e-commerce logistics," Journal of Kunming College for Ethnic Cadres, vol. 2, pp. 100-101, 2016.

[11] H. Feilong, H. Guan, and S. Wang, "The spatial distribution characteristics and influencing factors of the level of e-commerce development in Chinese cities," Economic Geography, vol. 36, no. 2, pp. 1-10, 2016.

[12] Y. Wu, "Discussion on the development trend of mobile e-commerce," Jiangsu Business Forum, vol. 31, pp. 45-46, 2016.

[13] J. Li, "The development status and future trends of China's e-commerce," Hebei Academic Journal, vol. 36, no. 1, pp. 107-109, 2016.

[14] Y. Liu, "Innovation and development trend of cross-border e-commerce logistics model," Modern Communication, vol. 3 , pp. 255-256, 2018.

[15] Z. Lang, "The innovation and development trend of crossborder e-commerce logistics model," Market Modernization, vol. 6, pp. 63-64, 2017.

[16] J. Wang, "Research on the training mode of cross-border E-commerce talents-taking Yiwu city as an example," Chinese Market, vol. 49, p. 153, 2016.

[17] B. Zhao, "Research on the development of China's crossborder E-commerce and government supervision: taking small cross-border online shopping as an example," Reform and Strategy, vol. 33, no. 1, pp. 133-135, 2017.

[18] Y. Du, "Research on the development strategy of cross-border e-commerce under the background of "one belt one road" initiative," Economic System Reform, vol. 6, pp. 66-70, 2016.

[19] W. Shen, "Analysis of e-commerce model," Taxation, vol. 17, no. 161, pp. 135-158, 2017.

[20] Y. Zhao, "The development model and strategy suggestion of my country's cross-border e-commerce," China Business Forum, vol. 4, pp. 28-29, 2018.

[21] X. Kong, "The development model and strategy suggestion of my country's cross-border e-commerce," Market Modernization, vol. 21, no. 858, pp. 49-50, 2017.

[22] X. Shen, "E-commerce development strategies for small and medium-sized enterprises," China Business Forum, vol. 18, pp. 13-14, 2017. 
[23] Y. Liu, "Problems and countermeasures for the development of cross-border e-commerce small trade," Information Construction, vol. 2, no. 213, p. 334, 2016.

[24] H. Qian and H. Jiang, "An empirical study on B2C crossborder E-commerce logistics mode selection," Business Research, vol. 12, pp. 118-125, 2016.

[25] X. Gong, "The development model and strategy suggestion of my country's cross-border e-commerce," Fortune Today, vol. 13, p. 56, 2017.

[26] K. Deng, "Exploration of the new normal mode of e-commerce in the belt and road strategic zone," Knowledge Economy, vol. 1, pp. 68-69, 2017.

[27] J. Wu, "Review of research on tourism E-commerce," Journal of Hubei Correspondence University, vol. 30, no. 1, pp. 83-84, 2017.

[28] J. Yang, "E-commerce in the "Internet +" era," Taxation, vol. 17, p. 133, 2017. 